\title{
Bildungsangebote als Bindeglied zwischen Forschung und Praxis - Wie aus Forschungsergebnissen zu neuen Technologien praxisrelevante Ideen für Innovationen entstehen
}

\author{
Jan N. Gremmel • Linda Grogorick • Susanne Robra-Bissantz • \\ David M. Woisetschläger
}

Eingegangen: 29. September 2021 / Angenommen: 20. Januar 2022 / Online publiziert: 14. Februar 2022 (C) Der/die Autor(en) 2022

Zusammenfassung Bildungsangebote für die Praxis sind ein wesentlicher Erfolgsfaktor dafür, technologischen Innovationen marktfähig zu machen. Sie sind eine Voraussetzung, um die unternehmerische Wettbewerbsfähigkeit auszubauen und neue Märkte zu erschließen. Bildungsinstitutionen sollten bei der Gestaltung von Bildungsangeboten das so genannte Wissensdreieck aus Bildung, Forschung und Praxis berücksichtigen und entsprechen Akteure aus Forschung und Wirtschaft involvieren, um von den wechselseitigen Verbindungen und Interaktionen zu profitieren. Der vorliegende Beitrag zeigt anhand des kooperativen Gestaltungsprozesses eines Bildungsangebots zur Grundlagentechnologie Blockchain im Kontext urbaner Mobilität auf, wie Bildung, Forschung und Praxis gemeinsam neues Wissen als Basis von Innovationen schaffen. Der Mehrwert unseres Beitrags liegt in der Erstellung eines übertragbaren Gestaltungsprozesses als Vorbild für zukünftige Bildungsangebotsgestaltungen.

Schlüsselwörter Wissensdreieck · Wissenstransfer · Bildungsangebote für Praxis · Blockchain · Urbane Mobilität

\footnotetext{
Jan N. Gremmel $(\varangle) \cdot$ David M. Woisetschläger

Lehrstuhl für Dienstleistungsmanagement, Technische Universität Braunschweig, Mühlenpfordtstraße 23, 38106 Braunschweig, Deutschland

E-Mail: j.gremmel@tu-braunschweig.de

Linda Grogorick · Susanne Robra-Bissantz

Lehrstuhl für Informationsmanagement, Technische Universität Braunschweig,

Mühlenpfordtstraße 23, 38106 Braunschweig, Deutschland

E-Mail: 1.grogorick@tu-braunschweig.de
} 


\title{
Educational Offers as a Link Between Research and Practice-How Research Findings on new Technologies give Rise to Practice-Relevant Ideas for Innovations
}

\begin{abstract}
Educational offers for industry are essential to foster a smooth transfer of new technologies and developments into marketable innovations. These offers support companies' improvements in their competitiveness and allow them to target new markets. Educational institutions should consider the knowledge triangle to include actors from research and industry to jointly design educational to benefit from the mutual connections and interactions. Using a collaborative design process of an educational offering for blockchain technology in the context of urban mobility, this paper demonstrates how research and practice work together to create new knowledge as the basis for innovation. The added value of our contribution lies in the creation of a transferable design process as a model for future educational service designs.
\end{abstract}

Keywords Knowledge Triangle $\cdot$ Knowledge Transfer $\cdot$ Educational Offers for Industry $\cdot$ Blockchain · Urban Mobility

\section{Einleitung}

Der Technologietransfer forscherischer Erkenntnisse in die Praxis ist ein altbekanntes, aber immer noch existentes Problem (Reddy und Zhao 1990; Phan et al. 2009; Miller et al. 2018). Dabei sind neue Technologien häufig die Basis der Innovation von Produkten oder Dienstleistungen. Gleichgültig jedoch, ob es sich um die Blockchain Technologie, das Internet of Things oder auch künstliche Intelligenz handelt - auf dem Weg von der oftmals technologie-zentrierten Forschung hin zur praxisrelevanten Innovation besteht häufig eine wesentliche Lücke darin, dass es weder den (technisch) Forschenden noch dem wirtschaftlich denkenden Unternehmen gelingt, die Potenziale der Technologie in Chancen für wirtschaftliche Innovationen zu übersetzen (Miller et al. 2018).

Zum Überwinden dieser Lücke kommt Institutionen insbesondere der Hochschulbildung eine besondere Rolle $\mathrm{zu}$, um Potenziale technologischer Innovationen zu prüfen und lösungsorientiert am Bedarf der Praxis zu vermitteln. Die Gestaltung von Bildungsangeboten für die Wirtschaft ist ein wesentlicher Mechanismus zur Ermöglichung eines Wissenstransfers, um neuartige Technologien in wirtschaftliche und gesellschaftliche Innovationen zu übersetzen. Dabei zeigen neuere Studien (z.B. Asarta et al. 2018), der Bertelsmann Stiftung (2017) oder des MIT (Sarma und Bonville 2020), dass insbesondere die Wissensvermittlung zu neuartigen Technologien ausbaufähig ist. Daher sollten Institutionen aus Bildung, Forschung und Wirtschaft gemeinsam in der Umsetzung neuartiger Technologien hin zu Innovationen für Wirtschaft und Gesellschaft agieren. Die Kollaboration kann in Form eines Wissensdreiecks beschrieben werden. Hierbei bilden die drei Institutionen jeweils die Eckpunkte des Dreiecks und produzieren durch wiederkehrende Interaktionen (Praxis)-Probleme sowie Lösungsansätze (Lassnigg et al. 2017). Eine besondere 
Form der Wissensvermittlung an die Praxis nehmen digitale Bildungsangebote ein, die unter anderem dafür geeignet sind Wissen sichtbarer zu machen und eine breitere Zielgruppe zu erreichen (Whitaker et al. 2016). Im Fallbeispiel dieses Beitrags handelt es sich um die Vermittlung von Wissen über eine neuartige Technologie. Entsprechend muss zunächst grundlegendes Wissen vermittelt und für die Potenziale sensibilisiert werden. Zur Wissensvermittlung wird im Fallbeispiel ein digitales Bildungsangebot konzipiert. Unabhängig davon, ob es sich um ein analoges oder digitales Bildungsangebot handelt, ist es essentiell, dass an diesen Bildungsangeboten Forschung und Praxis mitwirken. Hochschulen kommt dabei eine besondere Funktion zu, weil sie zum einen Teil der Forschung sind, zum anderen aber die Rolle der Bildung übernehmen und die Lücke zwischen Forschung und Praxis schließen können (Miller et al. 2018). Entsprechend fokussieren wir uns bei der Rolle der Bildung auf Hochschulen.

Den Austausch innerhalb des Wissensdreiecks zu verbessern, ist eine der wesentlichen Bestrebungen der Europäischen Kommission auf dem Weg zu einer wettbewerbsfähigen, wissensbasierten und folglich dynamischen Wirtschaft (EU Kommission 2005). Im Kern geht es darum, die Eckpunkte des Wissensdreiecks, d.h. die Institutionen, sowie deren Verbindungen und kontinuierlichen Interaktionen zu stärken, um Europa erfolgreicher darin zu machen, wissenschaftliche Fortschritte in marktfähige Innovationen zu überführen (Potočnik 2006). Wird dieser Herausforderung nicht ausreichend begegnet, so kann dies ein Grund für das häufig beklagte Versagen europäischer Länder bei der Umsetzung wissenschaftlicher Fortschritte in marktfähige Innovationen sein (Fragkandreas 2017).

An diesen Ausgangspunkt setzt dieser Beitrag an, um ein übertragbares Konzept dafür aufzuzeigen, wie aus aktuellen Forschungsbeiträgen zu einer bestimmten Technologie ein Bildungsangebot für die Praxis durch den Austausch mit der Praxis wird - um diese zu Innovationen zu befähigen. Im vorliegenden Beitrag wird dies am Beispiel der neuartigen Blockchain Technologie verdeutlicht, wobei der Prozess auch auf andere Technologien übertragbar ist. In diesem Beitrag skizzieren wir den Gestaltungsprozess eines digitalen Bildungsangebotes zu Blockchain-Anwendungsfällen im Kontext urbaner Mobilität. Diesem Prozess zu Grunde liegt das Wissensdreieck, in dem verschiedene Akteure aus Forschung und Praxis gemeinsam die Grundlage für neues Wissen als Basis von Innovation schaffen und dabei voneinander profitieren. Ziel ist es an einem konkreten Beispiel aufzuzeigen, wie mit dem Wissen zu einer Grundlagentechnologie wie der Blockchain ein anwendungsorientiertes, aber wissensgetriebenes Weiterbildungsangebot erstellt wird.

Dafür werden in den beiden folgenden Kapiteln zunächst die theoretischen Grundlagen für den Gestaltungsprozess entlang des Wissensdreiecks sowie der zu vermittelnden Blockchain-Technologie beschrieben. Dabei dient das Wissensdreieck als Basis für den Gestaltungsprozess. Darauf aufbauend folgt eine konkrete und praxisorientierte Beschreibung des Vorgehens zur Entwicklung des Gestaltungsprozesses und des finalen Lehrkonzepts des Bildungsangebots. Wir schließen mit einer Reflektion des Vorgehens sowie den Implikationen für Bildung, Forschung und Praxis. 


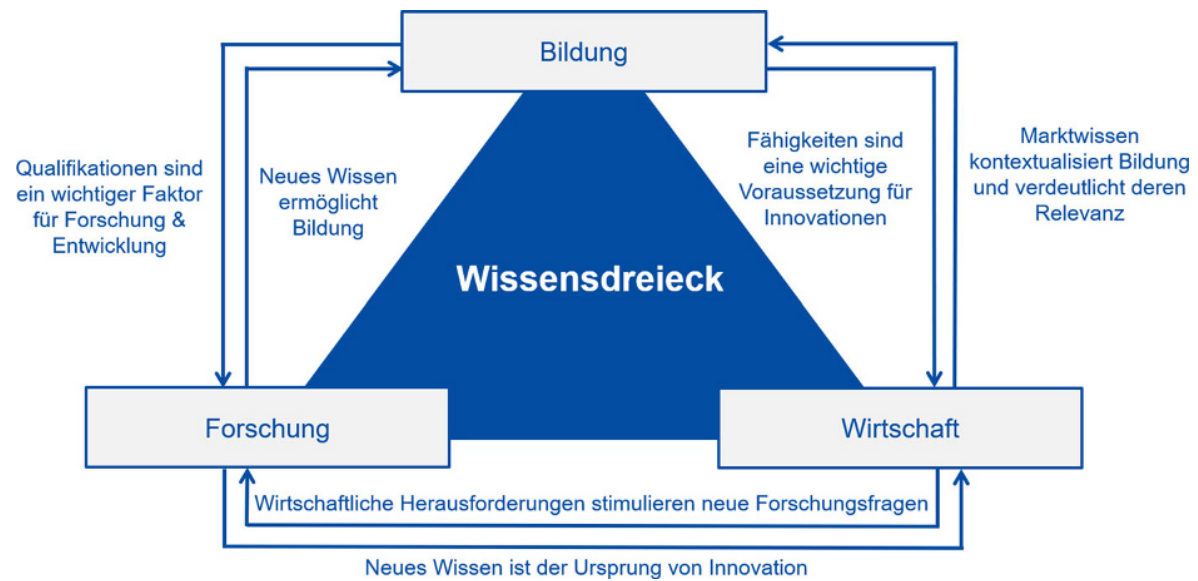

Abb. 1 Wissensdreieck (in Anlehnung an European Institute of Innovation and Technology (2012))

\section{Das Konzept des Wissensdreiecks}

Für das Konzept des Wissensdreiecks hat sich noch keine einheitliche Definition etabliert, da sich vorhandene Erklärungen in Bezug auf die Beschriftungen aber auch der anvisierten Ziele unterscheiden. Während im wissenschaftlichen Kontext mit dem Wissensdreieck die Interaktionen zwischen Bildung, Forschung und Innovation (Unger et al. 2020) gemeint ist, verwenden politische Kontexte häufig andere Bezeichnungen. Wie in Abb. 1 dargestellt wird vom Europäischen Innovations- und Technologieinstitut (EIT) damit die Interaktion zwischen Bildung, Forschung und Wirtschaft verstanden.

Beide Varianten des Wissensdreiecks beziehen sich auf die Schaffung von Wissen und dessen Anwendung durch Innovation. Einerseits dient das Konzept dem Beschreiben und Verstehen eines wissensintensiven Ökosystems, welches eine enge Zusammenarbeit (oder Integration nach dem Verständnis des EIT) der verschiedenen Akteure erfordert. Andererseits verweist das Konzept auf den umfassenderen Auftrag der Hochschulen in ihrer Doppelrolle von Forschung und Bildung die Innovationsfähigkeit der Wirtschaft zu erhöhen (Lassnigg et al. 2017). Unabhängig davon, welche Definition herangezogen wird, teilen sie jedoch die Kernaussage über eine zyklische Natur von Innovationen und die vielfältigen existierenden Input- und Feedbackschleifen zwischen den Akteuren eines Innovationssystems (EIT 2012).

Beispielsweise sind qualifizierte Arbeitskräfte die Voraussetzung für die Durchführung von Forschungs- und Entwicklungsvorhaben sowie für die Markteinführung neuer Produkte und Services. Die Qualifizierung muss durch wissensgetriebene und an dem aktuellen Forschungsstand ausgerichtete Bildungsprogramme vermittelt werden. Im Gegenzug sollten Wissen und aktuelle Marktentwicklungen eine Rückkopplung zu Bildungsprogrammen haben. In ähnlicher Weise ist neues Wissen der Ursprung für Innovationen, im Gegenzug entstehen im wirtschaftlichen Wettbewerb laufend neue Herausforderungen, die eine Inspiration für die Forschung darstellen (EIT 2012). 
Das Konzept des Wissensdreiecks bildet diese Zusammenhänge ab und verdeutlicht die gegenseitigen Vorteile, die aus dem engen Wechselspiel zwischen Bildung, Forschung und Wirtschaft entstehen können (Abb. 1). Anhand der Entwicklung eines Weiterbildungsangebots zu Blockchain wird in diesem Beitrag gezeigt, wie die Verbindung mittels eines digitalen Lernangebots verbessert werden kann.

\section{Blockchain im Kontext urbaner Mobilität}

Unter den Distributed-Ledger-Technologien gilt die Blockchain als eine der bekanntesten Architekturen zur Ausführung, Validierung und Speicherung von Transaktionen (Pilkington 2016). Im Gegensatz zu zentralen Netzwerkarchitekturen ist die Blockchain eine dezentrale Architektur basierend auf einem Peer-to-Peer-Netzwerk von Computern, welche alle gleichzeitig ein Blockchain-Protokoll ausführen (Meier und Stormer 2018). Das heißt, Transaktionen innerhalb von Blockchain-Netzwerken werden direkt zwischen den Teilnehmenden des Netzwerks ausgeführt, weshalb kein zentraler Server erforderlich ist und entsprechend kein Mittelsmann im Transaktionsprozess involviert ist (Pilkington 2016). Kumar et al. (2021) schreiben der Blockchain-Technologie das Potenzial zu, Transaktionen in Geschäftsbeziehungen sicher abzuwickeln. Somit ist die Blockchain-Technologie vor allem geeignet, um Vertrauen in entstehende Geschäftsbeziehungen aufzubauen oder um Daten in einem Netzwerk von Teilnehmenden automatisiert und sicher zu übermitteln (Halaburda et al. 2019). Essentielle Funktionen, die durch die Nutzung von Blockchain ermöglicht werden, sind der Einsatz von Smart Contracts, digitale Authentifizierung, eine neue Art der Datenkonnektivität und (Non-fungible) Token (Büttgen et al. 2021; Crosby et al. 2016; Schütz et al. 2018). Smart Contracts ermöglichen bspw. selbstauslösende Transaktionen, wenn vordefinierte Bedingungen erfüllt sind, während die digitale Authentifizierung über die Blockchain ermöglicht (personenbezogene) Daten auf einem Block zu speichern und im Zusammenspiel mit der Datenkonnektivität diese Daten mit anderen Teilnehmenden im Blockchain-Netzwerk zu teilen (Ante 2021). Token beschreiben nach Treiblmaier (2021) eine Werteinheit, die einen Wertgegenstand, ein spezielles Nutzungsrecht oder eine Zahlung repräsentiert (vgl. Oliveira et al. (2018) für eine aktuelle Auseinandersetzung mit (Non-fungible) Token).

Typische Anwendungsbereiche der Blockchain-Technologie liegen derzeit bspw. im Finanzsektor in der Anwendung in Form von Kryptowährungen oder zur Zahlungsabwicklung (Meier und Stormer 2018), in der industriellen Produktion (Bartsch et al. 2018) oder der Transformation von Städten in Smart Cities (Bagloee et al. 2021). Wesentlicher Bestandteil auf dem Weg zur Weiterentwicklung zu einer Smart City ist die Berücksichtigung der urbanen Mobilität. Im Feld der urbanen Mobilität wird das Potenzial der Blockchain-Technologie zunehmend von Start-Ups oder Großunternehmen wie IBM oder Daimler exploriert und in ersten Geschäftsfällen angewendet. Erfolgreiche Beispiele der Anwendung von Blockchain in der Mobilität ist etwa die Etablierung von Fahrzeugregistern durch Start-Ups wie carvertical. Hierbei werden Fahrzeuge in einer dezentralen Datenbank durch ihre Fahrgestellnummer verknüpft, sodass potenzielle Käuferinnen und Käufer sich über den Kilo- 
meterstand, eingetragene Mängel oder Unfälle informieren können. Abseits dieses Beispiels fehlt es der Blockchain-Technologie noch an Durchschlagskraft, sodass potenzielle Anwendungen eher auf dem Papier bestehen oder lokal getestet werden. Einen Erklärungsansatz dafür bietet der aktuelle Forschungsstand und der in der Unternehmenspraxis vorhandene aber ausbaufähige Wissensstand zur BlockchainTechnologie (Lacity und van Hoek 2021).

Der aktuelle Forschungsstand zu Blockchain zeigt, dass die Technologie stabil einsetzbar ist und bereit wäre in Geschäftsfällen angewendet zu werden. Jedoch ist aufgrund der relativen Neuheit der Technologie noch nicht hinreichend bekannt, was die Blockchain leisten bzw. nicht leisten kann. Dies ist jedoch ein kritischer Erfolgsfaktor, um potenzielle Anwendungsfälle der Blockchain-Technologie in erfolgreiche Business Cases zu transformieren (Lacity und van Hoek 2021). Entsprechend muss das Wissen zur Blockchain-Technologie an relevante Stakeholder weitervermittelt werden.

\section{Kooperativer Gestaltungsprozess des digitalen Bildungsangebots}

Für die Entwicklung des digitalen Weiterbildungsangebots zu Anwendungsfällen der Blockchain im Kontext urbaner Mobilität arbeiten Akteure aus Bildung, Forschung und Wirtschaft in einem kooperativen Gestaltungsprozess zusammen. Dieser ist in Abb. 2 visualisiert. Im Kontext der urbanen Mobilität kommt mit den Städten noch

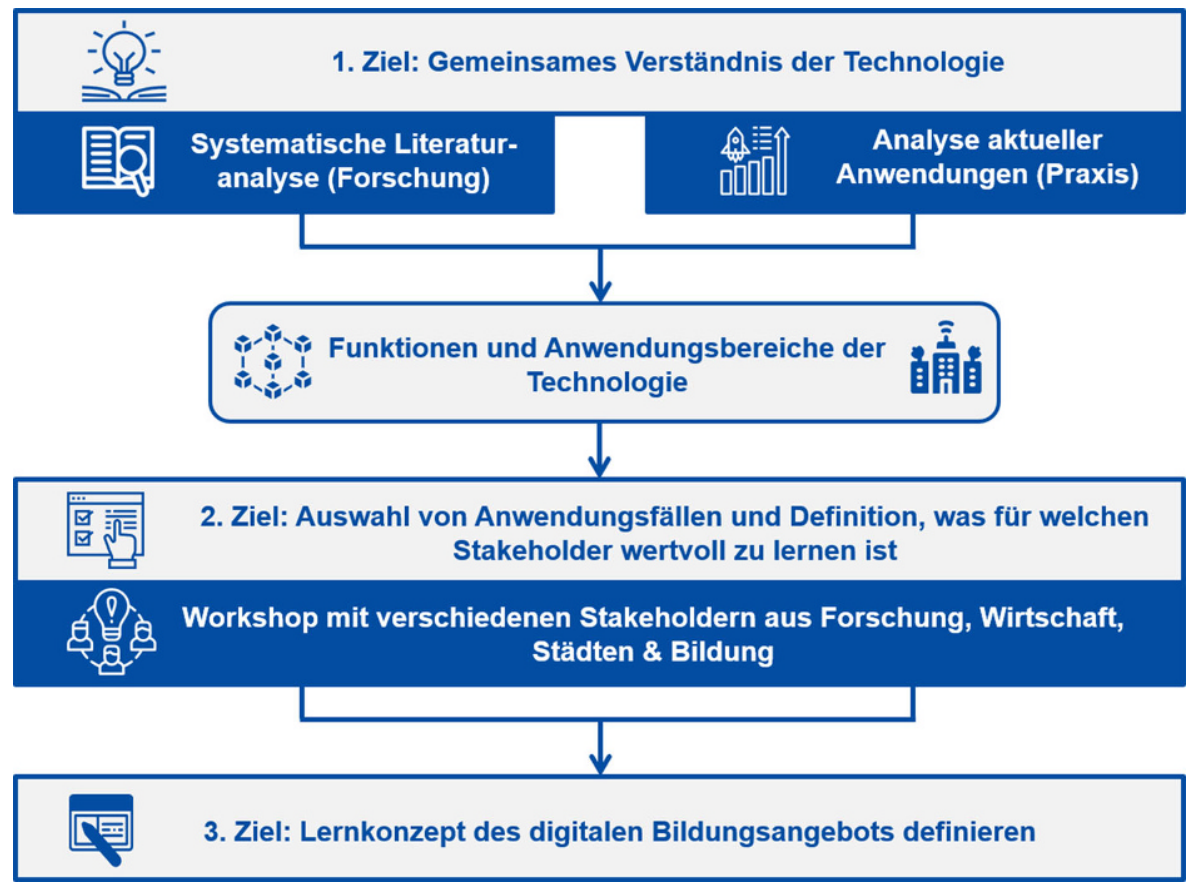

Abb. 2 Kooperativer Gestaltungsprozess des digitalen Bildungsangebots 
ein weiterer essentieller institutioneller Vertreter hinzu, der im Wissensdreieck im Bereich der Wirtschaft einzuordnen ist, jedoch zusätzlich noch mit der Privatwirtschaft interagiert.

Zunächst ist ein gemeinsames Verständnis von Blockchain in urbaner Mobilität zwischen den verschiedenen Akteuren aus Bildung, Forschung und Wirtschaft erforderlich. Dazu führt die das Bildungsprogramm anbietende Bildungsinstitution in einem ersten Schritt eine systematische Literaturanalyse zur Erfassung des aktuellen Forschungsstands in Kombination mit einer Praxisanalyse aktueller Anwendungen zur Identifikation existierender Lösungen in der Wirtschaft durch. Die systematische Literaturanalyse zur Blockchain-Technologie im Allgemeinen und spezifischen zu potenziellen Anwendungsbereichen im Kontext der urbanen Mobilität wurde genutzt, um ein grundsätzliches Verständnis der Technologie aufzubauen und Funktionen zu identifizieren. Die Praxisanalyse wird dazu genutzt, um tiefere Informationen über aktuelle Trends in Bezug auf die Blockchain-Technologie zu erhalten, die in der Literatur nicht behandelt werden, sowie Beispiele für die Anwendung der Blockchain-Technologie in der realen Welt zu identifizieren. Somit dient der initiale Schritt der inhaltlichen Ausgestaltung der Workshops und des Bildungsangebots, um Funktionen und Anwendungsbereiche von Blockchain allgemein und im konkreten Anwendungskontext der urbanen Mobilität zu erfassen sowie strukturiert aufzuarbeiten. Mit dieser Suchstrategie war es möglich, einen systematischen Ansatz für die Dokumentation von Anwendungsfällen und Potenzialen der Blockchain $\mathrm{zu}$ entwickeln.

Im zweiten Schritt werden in gemeinsamen Workshops mit Stakeholdern aus Wirtschaft und Forschung Anforderungen an ein digitales Weiterbildungsangebot exploriert. Dafür werden Funktionen und Anwendungsbereiche der BlockchainTechnologie in der urbanen Mobilität aus der initialen Recherche abgeleitet und als Grundlage für die Workshops verwendet. Ziel der Workshops ist die Auswahl von Blockchain-Anwendungsfällen, als auch die Definition dessen, was für welche Stakeholder wertvoll zu lernen ist. Darauf aufbauend wird im dritten Schritt das Lernkonzept des digitalen Bildungsangebots erstellt, welches die praktischen Anforderungen und Probleme mit den definierten Anwendungsfällen in der urbanen Mobilität widerspiegelt, bei denen die Blockchain-Technologie potenziell helfen könnte.

Nachdem der kooperative Gestaltungsprozess des digitalen Bildungsangebots allgemein erläutert wurde, sind in den nachfolgenden Abschnitten die Details der einzelnen Schritte beschrieben.

\subsection{Literatur- und Praxisanalyse als Ausgangspunkt}

Die für den ersten Schritt notwendige systematische Literaturrecherche beschreibt eine Methode, um wissenschaftliche Studien und Berichte zuverlässig und präzise zusammenzufassen. Die systematische Literaturrecherche wurde im Fallbeispiel nach Moher et al. (2010) durchgeführt. Für diese systematische Überprüfung des Forschungsstandes wurden sechs wissenschaftliche Literaturdatenbanken (AIS eLibrary, ScienceDirect, ACM Digital Library, Scopus, IEEE XPlore, Taylor \& Francis) abgefragt, um sicherzustellen, dass ein breites Spektrum an Fachgebieten, wie z. B. 
Informatik oder Wirtschaftswissenschaften, abgedeckt wird. Insgesamt lieferten die Literaturdatenbanken im Fallbeispiel 2029 Ergebnisse. Diese wurden in eine Datenbank übernommen und durch eine systematische Filterung auf 40 Artikel reduziert. Hierzu wurden bspw. doppelte Artikel oder Artikel aus anderen Disziplinen entfernt. Auf Grund des besonderen Fokus auf urbane Mobilität im Fallbeispiel wurden die verbliebenen Artikel nach ihrem Kontext gefiltert. Diese 40 Artikel wurden mit besonderem Fokus auf die vorgestellten Anwendungsfälle, die Blockchain-fähigen Funktionen und die genutzten Blockchain-Potenziale in den verschiedenen Anwendungsfällen analysiert. In den 40 analysierten Artikeln konnten verschiedene Basisanwendungsfälle (z. B. Transportmanagement) identifiziert werden, die aus mehreren untergeordneten Anwendungsfällen (z. B. Priorisierung im Verkehr oder Profiling des Fahrverhaltens) bestehen.

Die Literaturrecherche hat nicht nur gezeigt, welche Anwendungsfälle von Blockchain, Blockchain-fähigen Funktionen (Smart Contract, digitale Authentifizierung, Datenkonnektivität \& Datenaustausch und (nicht-fungible) Token) und Potenziale (Automatisierung, Vertrauen, Transparenz, Datensicherheit, Geschwindigkeit, Einnahmequelle, Investition) existieren. Vor allem wurde aufgezeigt, welche Anwendungsfälle welche Funktionen nutzen und welche Potenziale der Blockchain für welche Anwendungsfälle besonders relevant sind.

Komplementär zur Literaturanalyse wurde eine Praxisanalyse durchgeführt, um die Erkenntnisse aus der systematischen Literaturrecherche $\mathrm{zu}$ ergänzen und $\mathrm{zu}$ unterstützen. Ziel der Praxisanalyse war es im Fallbeispiel, die Erkenntnisse über aktuelle und zukünftige Trends bei der Anwendung der Blockchain-Technologie in der Wirtschaft, die in der Literatur nicht behandelt wurden, zu vertiefen. Die Praxisanalyse im vorliegenden Anwendungsfall erfolgte semi-strukturiert in Anlehnung an Nagel et al. (2019). Zunächst wurde systematisch in relevanten Datenbanken nach Unternehmen gesucht. Da es sich im vorliegenden Fallbeispiel um die Anwendung von Blockchain-Technologie in Europa handelt, wurden entsprechend Unternehmen aus dem Euro Stoxx 50 Index betrachtet. Darüber hinaus wurde die chaineurope Datenbank abgefragt, welche eine Übersicht über aktuelle Blockchain-Start-Ups in Europa bietet. Start-Ups sind dafür bekannt, dass sie neuartige Technologien als erstes in Produkte und Services überführen (Weiblen und Chesbrough 2015), von daher war es wenig überraschend, dass insbesondere Start-Ups die Technologie nutzen. Eine Besonderheit wurde für das vorliegende Fallbeispiel aber festgestellt, denn die betrachteten Start-Ups fokussieren sich oftmals auf wenige spezifische Funktionen, um einen Geschäftsfall abzudecken. Daher waren diese besonders geeignet, um die Ergebnisse der Literaturanalyse zu vervollständigen. Ergänzt wurde die strukturierte Suche durch gezielte Schlagwortsuchen, Nachrichten und Reports in Anlehnung an eine gezielte Stichprobenstrategie (Patton 1990). Die Praxisanalyse unterstützt die Erkenntnisse aus der Literaturanalyse, da sie im Fallbeispiel Belege für bestimmte Eigenschaften der Blockchain-Technologie oder ihrer Anwendung liefert.

Schließlich lieferte die Praxisanalyse ein ganzheitliches Bild für die systematische Dokumentation von Anwendungsfällen als breitere Basis für die Workshops, da sie es ermöglicht zu überprüfen, ob die in den Workshops entwickelten Anwendungsfälle neu und nicht existent sind, bereits in der Literatur thematisiert oder auch bereits als Geschäftsfall von einem Start-Up aufgesetzt wurden. 
Insgesamt bildeten die Erkenntnisse des zweistufigen Ansatzes die inhaltliche Grundlage für explorierende Workshops mit Partnern aus Praxis und Forschung.

\subsection{Gemeinsamer Workshop mit der Industrie in Kombination mit lokalspezifischen Workshops mit Städten}

Aus der Recherche zum aktuellen Forschungsstand zur Blockchain-Technologie ging hervor, dass die Technologie spezifiziert ist, es aktuell aber insbesondere auf der Praxisseite an den notwendigen Kenntnissen des Nutzens und zur Nutzung der Technologie fehlt (Lacity und van Hoek 2021). Daher ist eine Wissensvermittlung an diese Akteure notwendig. Unter Bezugnahme des Wissensdreiecks kommen diese Akteure aus der Forschung und Wirtschaft. Jedoch blieb hierbei unklar, welche Akteure aus der Wirtschaft angesprochen werden sollten. Erkenntnisse dazu liefert die systematische Literatur- und die Praxisanalyse, wonach die Wirtschaftsakteure mit Bedarf an Wissensvermittlung aus der Industrie, aber vor allem aus der Stadtverwaltung kommen. Insbesondere die Stadtverwaltung nimmt eine Schlüsselrolle als regulatorischer Ermöglicher der Technologie ein.

Somit dienten die Workshops als Austauschort zwischen Akteuren aus der Bildung, Forschung, Industrie und Stadtverwaltung, wobei der Workshop vom Bildungsinstitut initiiert werden sollte. Die Workshops verfolgten das übergeordnete Ziel, durch Interaktion zwischen Beteiligten des Wissensdreiecks den Bedarf und die Anforderungen an ein Bildungsangebot an die Praxis zu explorieren, um darauf aufbauend das digitale Bildungsangebot entsprechend der Bedürfnisse aufzustellen, aber auch, um zu erkennen, wie die erkannten Funktionen effektiv vermittelt werden können.

Ein erster Workshop wurde im Fallbeispiel bewusst mit Akteuren aus Forschung und Industrie initiiert. Zum einen, um das übergeordnete Ziel der Bedarfsermittlung zu verfolgen, zum anderen aber auch, um die eigenen Erkenntnisse, die vor allem auf einer Sekundäranalyse basieren, mit Experten aus dem Bereich Blockchain abzugleichen. Daher wurden zum Workshop unter anderem Experten eingeladen, die sich in ihrer Forschung oder in der Praxis mit der Blockchain-Technologie beschäftigen. Insgesamt haben am Workshop 18 Personen aus 10 verschiedenen Organisationen teilgenommen. Der Workshop wurde online per Videokonferenz und unter der Verwendung der online Kollaborationsplattform Mural durchgeführt. In Abb. 3 ist der Verlauf des Workshops abgebildet. Der Workshop dauerte zwei Stunden und begann mit einem Warm-Up zur Vorstellung des Workshops und seiner Ziele sowie einem gegenseitigen Kennenlernen. Der „Ice-Breaker“ sollte die Gesprächsatmo-

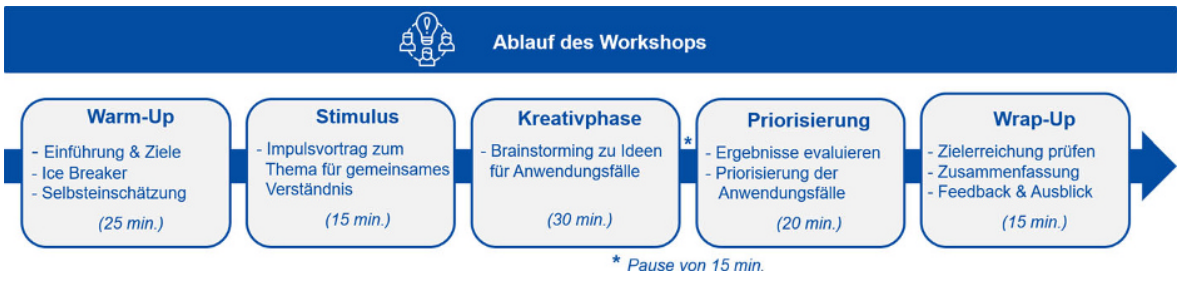

Abb. 3 Gemeinsamer Workshop zur Bedarfserhebung des Bildungsangebots 
sphäre auflockern. Zum Abschluss dieser Phase sollten sich die Teilnehmenden selbst zu ihren Vorkenntnissen zur Technologie einschätzen und kurz ihre Entscheidung begründen. Hierbei zeigte sich, dass das eigene Wissen zur Technologie relativ höher eingeschätzt wurde als die persönlichen Berührungspunkte mit der Technologie. Insgesamt wurde das eigene Vorwissen aber insbesondere von Teilnehmenden aus der Praxis als ,niedrig“ eingestuft. Dies spiegelt die Zusammensetzung der Gruppe wider sowie die geringe Verbreitung der Technologie in der Praxis. Im Anschluss an das Warm-Up folgte ein kurzer Stimulusvortrag. Der Stimulusvortrag basierte auf den zusammengetragenen Erkenntnissen aus der Literatur- und Praxisanalyse. Ziel des Vortrags war es, ein gemeinsames Verständnis zur Technologie aufzubauen. Dies ist essentiell für die sich anschließende kreative Phase. In dieser Kreativphase sollten die Teilnehmenden ihr vermitteltes Wissen nutzen, um Anwendungsfälle zu entwickeln. Somit verfolgt die Kreativphase zwei Ziele: Implizit wird durch den Vortrag die Klarheit der Wissensvermittlung geprüft, wobei Rückfragen als Ausgangspunkt für Verbesserungen genutzt werden können. Explizit wird das vermittelte Wissen direkt angewendet, wobei sich auch hier zeigt, wie klar das Wissen im Vortrag kommuniziert wurde. Wichtig ist es, dass in der Kreativphase eine Kreativmethode gewählt wird, die an die Gruppengröße sowie den Teilnehmenden ausgerichtet ist. Für den durchgeführten Workshop im Fallbeispiel wurde ein Brainstorming unter Verwendung der 1-2-4-All-Methode (Lipmanowicz und McCandless 2014) durchgeführt. Der Vorteil dieser Methode ist es, dass zu Beginn des Brainstormings alle Teilnehmenden alleine über den Sachverhalt nachdenken und in den darauffolgenden Iterationsrunden zunächst zu zweit und dann in einer vierer Gruppe Ideen gemeinsam weiterentwickeln, sodass zum Ende des Brainstormings konkrete Ideen im Plenum diskutiert werden können. Nach einer kurzen Pause wurden die Ergebnisse gemeinsam hinsichtlich des zu erwartenden Potenzials für Gesellschaft, Wirtschaft, Stadt und Bürgerinnen sowie Bürger priorisiert. Zum Abschluss wurde erneut eine Selbsteinschätzung zum Kenntnisstand über Blockchain initiiert, offene Fragen aufgenommen und über Trainingsbedarf diskutiert. Besondere Bedeutung kam hierbei der Behandlung des Datenschutzes bei. Darüber hinaus zeigte etwa die Selbsteinschätzung des Kenntnisstandes über Blockchain eine leichte Verbesserung bei Teilnehmenden aus der Praxis, wobei dies auch eine Momentaufnahme als Resultat der Auseinandersetzung im Workshop sein könnte.

Aufbauend auf den Erkenntnissen des ersten Workshops wurden vertiefende Workshops mit Akteuren aus Städteverwaltungen durchgeführt. Im Unterschied zu den Workshops mit Akteuren aus Forschung und Industrie waren die Workshops mit den städtischen Partnern auf die spezifischen Bedürfnisse und Anforderungen vor Ort ausgerichtet. Dies ist besonders relevant, da die Umsetzung von Anwendungsfällen in einem städtischen Kontext in hohem Maße mit den lokal-spezifischen Bedingungen zusammenhängt. Daher wurde eine Reihe von Workshops durchgeführt, wobei für einen Workshop jeweils Vertreter einer einzelnen Stadt eingeladen wurden. Die Struktur und der Inhalt der vertiefenden Workshops mit Städtepartnern ähnelten dem initialen Workshop, jedoch mit einem stärkeren Fokus auf städtespezifische Herausforderungen und weiteren Trainingsbedarf.

Insgesamt dienten die Workshops zur Ableitung von Anforderungen an ein Weiterbildungsangebot sowie den spezifischen Bedürfnissen der einzelnen Stakeholder. 
Eine Kernerkenntnis ist es bspw., dass die meisten Befragten zwar Blockchain-Technologie mit Kryptowährungen in Verbindung bringen, jedoch darüber hinaus keine Vorstellung darüber haben, was Blockchain leisten bzw. nicht leisten kann. Um das Potenzial in Business Cases aber voll verstehen und entfalten zu können, ist es notwendig eine grundlegende Vorstellung über die Technologie zu haben. Übertragen auf das Lernkonzept für das Bildungsangebot bedeutet dies, dass es zunächst erforderlich ist ein gemeinsames Grundwissen zu etablieren. Darauf aufbauend können dann Business Cases vermittelt werden, um das Potenzial der Technologie weiter zu verdeutlichen.

Somit schlagen die Workshops die Brücke zwischen Forschung und Wirtschaft. Die Workshops erfüllen für die Erstellung eines Bildungsangebots die Funktion, eine Interaktion zwischen beiden Akteursgruppen zu ermöglichen, um dabei das Potenzial der Technologie aufzuzeigen und gleichzeitig die Herausforderungen sowie Bedürfnisse bei der Anwendung der Technologie zu explorieren.

\subsection{Lernkonzept des Bildungsangebots}

Basierend auf den Erkenntnissen aus der Literatur- und Praxisanalyse sowie den Workshops mit Stakeholdern aus Wirtschaft und Forschung, wurde das Lernkonzept für das Bildungsangebot erstellt. Die digitalen Lernangebote wurden als interaktive Lerneinheiten entwickelt, die realistische und realisierbare Anwendungsfälle von Blockchain im Kontext urbaner Mobilität repräsentieren. Diese Anwendungsfälle basieren auf den zuvor durchgeführten Workshops. Eine Lerneinheit wird als nanoMooc angeboten, was einem audiovisuellen Format des digitalen Trainings entspricht. Die Bezeichnung nanoMooc ist ein im Rahmen des Programms geschaffener Neologismus für einen „massive open online course“ (MOOC) mit einem kurzen, fokussierten Training auf eine einzelne Kompetenz (nano), im vorliegenden Fall auf verschiedene Aspekte der Blockchain-Technologie. Entsprechend handelt es sich bei den nanoMoocs um eine reduzierte Form von MOOCs (vgl. Whitaker et al. (2016) für eine aktuelle Auseinandersetzung mit MOOCs), wobei im Wesentlichen die Kollaboration zwischen Teilnehmenden entfällt und die Wissensvermittlung im Vordergrund steht. Dennoch sind die einzelnen nanoMoocs in einer zusammenhängenden Kursstruktur eingebunden.

Um den Bedürfnissen der Teilnehmenden aus den Workshops zu entsprechen, bestehen diese aus zwei Kursstufen:

- Basis nanoMooc (mit Schwerpunkt auf Definition von Blockchain, Transaktionen in einem Blockchain-Netzwerk und Funktionen) ${ }^{1}$

- Use Case nanoMoocs (jeweils für einen spezifischen Anwendungsfall, der von verschiedenen Blockchain Funktionen profitiert)

Das übergeordnete Ziel der nanoMoocs ist es, den Lernenden als Grundlage zu dienen, um eigene Anwendungsfälle bzw. Geschäftsfälle durch eine Neukombination der Blockchain-fähigen Funktionen zu entwickeln, so dass Innovationen ent-

\footnotetext{
${ }^{1}$ Die nanoMoocs sind abrufbar unter: https://nanomoocs.cat/courses/course-v1:EIT_Urban_Mobility+ EITUM01+2021/about.
} 
stehen. Entsprechend absolvieren die Teilnehmenden zunächst den Basis nanoMooc zum Aufbau eines grundlegenden Wissens über die Blockchain-Technologie. Hier werden bspw. auch die Blockchain-fähigen Funktionen (Smart Contract, digitale Authentifizierung, Datenkonnektivität und Datenaustausch sowie (Non-fungible) Token) eingeführt. Im Anschluss können die Teilnehmenden aus spezifischen Use Case nanoMoocs wählen, die jeweils einen Anwendungsfall behandeln. Dabei werden die zuvor eingeführten Funktionen genutzt, um den Anwendungsfall zu beleuchten. Die gewählte Struktur erlaubt durch eine Modularität den Kurs durch neue nanoMoocs zu erweitern. Zu Beginn sollen vier Use Case nanoMoocs zur Verfügung stehen. Diese basieren auf den zuvor durchgeführten Workshops und spiegeln somit aktuell interessante Anwendungsfälle wider, wie bspw. die Möglichkeit einer ,Vehicle ID“. Diese erlaubt es Logistikunternehmen Vehikel und Ladeinformationen auf einer Blockchain zu verknüpfen und somit Informationen automatisch an Partner entlang der Lieferkette zu teilen, etwa wenn das Vehikel einen Grenzkontrollpunkt passiert. Da sich die Blockchain-Technologie noch in einer entstehenden Phase befindet (Lacity und van Hoek 2021), können in Zukunft weitere Anwendungsmöglichkeiten entstehen. Diese können dann durch zusätzliche nanoMoocs ergänzt werden.

Nachdem die Inhalte basierend auf den Ergebnissen der Workshops entschieden wurden, wurde die Struktur der Wissensvermittlung und -abfrage der einzelnen nanoMoocs bestimmt. Diese folgt der Lerntheorie des Constructive Alignment, die vorschlägt Lernziele, Lehr-/Lernaktivitäten und die Überprüfung des Erlernten aufeinander abzustimmen (Biggs und Tang 2011). Einzelne nanoMoocs können unterschiedlich gestaltet sein (z.B. mit überraschenden Elementen), folgen aber dennoch einem identischen Aufbau, der in Abb. 4 visualisiert ist. Jedes nanoMooc ist in drei Abschnitte unterteilt: Einführung, Haupt-Lehr-/Lernaktivitäten und Abschluss.

In der Einführung beginnt jedes nanoMooc mit einem Thought Starter, um das Interesse zu wecken. Thought Starter sind kleine Denkanstöße und können bspw. entweder ein anschauliches Beispiel oder ein paar Fakten und Statistiken sein. Hier ist es wichtig, Kreativität zu zeigen und das Interesse der Lernenden an der Lerneinheit zu wecken. Darüber hinaus sollten die Lernziele so formuliert werden, dass sie den Inhalt und das kognitive Niveau des zu lehrenden und zu lernenden Inhalts genau beschreiben. Die Lernziele sollten widerspiegeln, warum der Inhalt für die Lernenden wichtig ist. Um Lernziele zu definieren, ist die Orientierung an der Lernergebnis-Taxonomie von Anderson und Krathwohl (2001) mit den sechs kognitiven Prozessdimensionen (Erinnern, Verstehen, Anwenden, Analysieren, Evaluieren und Kreieren) und den vier Wissensdimensionen (faktisch, konzeptionell, prozedural und metakognitiv), von einfach bis komplex, empfehlenswert.

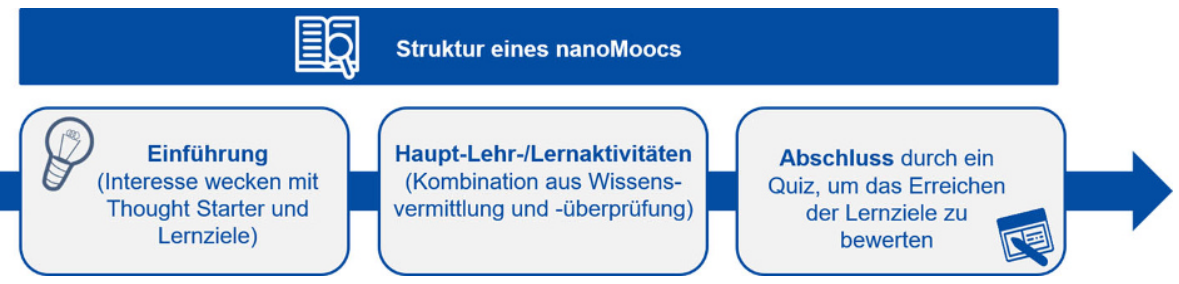

Abb. 4 Struktur eines nanoMoocs nach Lerntheorie des Constructive Alignment 
Im Anschluss an die Einführung mit dem Thought Starter und den definierten Lernzielen folgen die Haupt-Lehr-/Lernaktivitäten. Diese sind in verschiedene kleine Kapitel unterteilt, wobei jedes sowohl eine Wissensvermittlung als auch -überprüfung enthält. Beides sollte variiert werden, um eine höhere Akzeptanz der nanoMoocs zu erreichen und um Langeweile während des Lernprozesses zu vermeiden, so dass Lernende auch wirklich mit den nanoMoocs lernen wollen. Lernende eignen sich unterschiedliche Kenntnisse und Fähigkeiten an (z.B. in Präsentationen, Videos, Podcasts, Best-Practice-Start-Ups oder wissenschaftlichen Beiträgen) und wenden ihr dabei erworbenes Wissen in zu lösenden Aufgaben an. Die Aufgaben variieren von Ja-/Nein-Fragen, Single-/Multiple-Choice-Aufgaben, Markierungsaufgaben, Sequenzaufgaben (Drag \& Drop), Zuordnungsaufgaben (d.h. Zuordnung von Begriffen oder Bildern), Kreuzworträtseln und Textaufgaben (Lückentexte oder freie Texte). Es ist nicht notwendig, alle denkbaren Aufgabentypen einzubeziehen, sondern sicherzustellen, dass eine Vielzahl von ihnen verwendet wird und sie so gestaltet sind, dass das Erreichen der Lernziele überprüft werden kann (Kibler und Eckardt 2018).

Den Abschluss eines jeden nanoMoocs bildet ein finales Quiz, mit dem das Erreichen der in der Einführung formulierten übergeordneten Lernziele bewertet wird. Während in den Haupt-Lehr-/Lernaktivitäten auch Wissenstests integriert sind, um das in den einzelnen kleinen Kapiteln erworbene Wissen zu überprüfen, konzentriert sich der Abschlusstest auf die Überprüfung des Erreichens der allgemeinen Lernziele über alle Kapitel hinweg. Die verwendeten Aufgabentypen zur Überprüfung der Lernziele sollten ebenfalls variieren, wie im obigen Abschnitt beschrieben. Zusätzlich zum Quiz können am Ende Querverweise auf weitere Lerninhalte, die nicht Teil des nanoMoocs sind, aufgelistet werden, um das Interesse an weiteren Lernaktivitäten zu wecken und als Cliffhanger zu anderen verbundenen Lerninhalten zu dienen.

Die klar definierte Struktur jedes nanoMoocs soll es den Lernenden ermöglichen, sich voll und ganz auf Blockchain und die damit verbundenen Funktionen sowie die vorgestellten Anwendungsfälle in der urbanen Mobilität zu konzentrieren, so dass sie nach dem Lernprozess in der Lage sind, eigene Blockchain-Anwendungsfälle zu entwickeln, um ihr Innovationspotenzial zu stärken.

\section{Schlussbemerkungen}

Die Verbindungen und kontinuierlichen Interaktionen zwischen den verschiedenen Akteuren aus Bildung, Forschung und Wirtschaft gemeinsam entlang des Wissensdreiecks haben die Gestaltung eines digitalen Bildungsangebots zu Blockchain-Anwendungsfällen in der urbanen Mobilität ermöglicht. Dieser Gestaltungsprozess bietet ein übertragbares Konzept zur Etablierung neuer Weiterbildungsangebote in drei Schritten. Erstens ist die Durchführung einer systematischen Literaturanalyse zur Erfassung aktueller Entwicklungen aus der Perspektive der Forschung in Verbindung mit einer Praxisanalyse zur Identifikation der bereits vorhandenen praktischen Anwendung der Forschungsergebnisse notwendig, um einen Überblick sowohl aus Sicht der Forschung als auch Praxis zu bekommen. Im zweiten Schritt sind Work- 
shops mit Stakeholdern aus Forschung und Praxis $\mathrm{zu}$ initiieren, um Bedürfnisse und Anforderungen an ein Bildungsangebot zu ermitteln. Diese Workshops dienen als wichtiges Interaktionselement zwischen Bildung, Forschung und Wirtschaft, um die aktuell vorhandene Wissensbasis zu Technologien und Entwicklungen an den tatsächlichen Praxisbedarfen auszurichten und neues Wissen anschließend entsprechend der Bedürfnisse der Praxis zu vermitteln. Die vorgenommene Zweiteilung der Workshops hat sich für das vorliegende Projekt als erfolgreich herausgestellt, weil so genauer auf lokal-spezifische Anforderungen und Bedürfnisse der verschiedenen Stakeholder eingegangen werden konnte. Jedoch ist dies nicht immer notwendig, bspw. wenn keine lokal-spezifischen Unterschiede zu erwarten sind. Im dritten und abschließenden Schritt steht die Entwicklung des Weiterbildungsangebots im Mittelpunkt, was die Erstellung eines Lernkonzepts einschließt, welches durch eine klare Struktur lernförderlich ist.

Trotz des Potenzials des Gestaltungsprozesses erkennen wir einige Limitationen an unseren Erkenntnissen, die einen Ausgangspunkt für weitere Forschung bieten. Zunächst konnten wir den vorgestellten Gestaltungsprozess lediglich am Beispiel der Blockchain-Technologie mit besonderem Fokus auf urbane Mobilität testen. Daher ist es möglich, dass der Gestaltungsprozess durch die besonderen Eigenschaften der Blockchain-Technologie und der assoziierten Wissensvermittlung dieser speziellen Technologie bedingt ist. Wir ermutigen Bildungsinstitutionen ebenso wie Praktiker den Gestaltungsprozess in anderen Kontexten zu erproben und darüber hinaus auf andere neuartige Technologien zu übertragen, um eine Allgemeingültigkeit zu fördern und so die Wirkung der Erkenntnisse zu erhöhen. Weiterhin kam uns die Bereitschaft von Praxispartnern entgegen, an unseren Workshops teilzunehmen. Auch wenn wir relativ mehr Absagen als Zusagen erhalten haben, war es dennoch möglich mehrere Workshops durchzuführen. Dies ist nicht immer der Fall, dennoch möchten wir zukünftige Forschenden aufrufen sich nicht durch Absagen potenzieller Partner entmutigen zu lassen. Als erfolgsversprechend hat es sich im Rahmen dieses Projekts erwiesen, die Workshops digital durchzuführen und somit die verbundenen Reisekosten (bspw. durch An- und Abfahrt, Übernachtung) zu minimieren. Darüber hinaus hatten wir insbesondere für den initialen Workshop einen knappen Zeitrahmen definiert, um ebenfalls den oftmals vollen Terminkalendern der Praxispartner entgegenzukommen. Der Zeitrahmen wurde durch eine stringente Struktur aufgefangen. Dennoch war ein wesentliches Feedback der Teilnehmenden, dass sie sich mehr Zeit für den Workshop gewünscht hätten. Dem sollte bei der Planung weiterer Workshops, wenn möglich, entsprochen werden.

Institutionen der Hochschulbildung nehmen im Gestaltungsprozess eine Schlüsselrolle ein. Sie übernehmen die initiierende sowie konzipierende Funktion und schlagen somit die Brücke zwischen Forschung und Praxis. Um auf lange Sicht erfolgreicher dabei zu werden, Forschungserkenntnisse in marktfähige Innovationen zu überführen, liegt es sowohl an Forschung als auch Praxis Initiative zu ergreifen. Einerseits sollte die Forschung neben der Schaffung wissenschaftlicher Fortschritte häufiger den Kontakt zur Praxis suchen, nicht nur, um gemeinsame Forschungsprojekte voranzutreiben, sondern auch, um anschließend neu gewonnenes Wissen und Erkenntnisse in Weiterbildungsangebote für die Praxis zu überführen. Andererseits sollte die Praxis solchen „Aufrufen“, zum Beispiel für die Teilnahme an 
Workshops zur Förderung entsprechender Verbindungen und Interaktionen, folgen und diese als Chance wahrnehmen, um Bildungsangebote speziell für die eigenen Bedürfnisse entwickeln zu lassen. Beides kann im Sinne des Wissensdreiecks dazu beitragen neues Wissen zu schaffen und dessen Anwendung durch Innovation zu fördern, sodass alle Akteure des Wissensdreiecks voneinander profitieren.

Danksagung Dieser Artikel wurde im Rahmen des Projekts „Blockchain for Urban Mobility“ unter der Projektträgerschaft des European Institute of Innovation and Technology Urban Mobility (EIT UM) erarbeitet und mit Mitteln aus dem Business Plan 2021 gefördert.

Funding Open Access funding enabled and organized by Projekt DEAL.

Open Access Dieser Artikel wird unter der Creative Commons Namensnennung 4.0 International Lizenz veröffentlicht, welche die Nutzung, Vervielfältigung, Bearbeitung, Verbreitung und Wiedergabe in jeglichem Medium und Format erlaubt, sofern Sie den/die ursprünglichen Autor(en) und die Quelle ordnungsgemäß nennen, einen Link zur Creative Commons Lizenz beifügen und angeben, ob Änderungen vorgenommen wurden.

Die in diesem Artikel enthaltenen Bilder und sonstiges Drittmaterial unterliegen ebenfalls der genannten Creative Commons Lizenz, sofern sich aus der Abbildungslegende nichts anderes ergibt. Sofern das betreffende Material nicht unter der genannten Creative Commons Lizenz steht und die betreffende Handlung nicht nach gesetzlichen Vorschriften erlaubt ist, ist für die oben aufgeführten Weiterverwendungen des Materials die Einwilligung des jeweiligen Rechteinhabers einzuholen.

Weitere Details zur Lizenz entnehmen Sie bitte der Lizenzinformation auf http://creativecommons.org/ licenses/by/4.0/deed.de.

\section{Literatur}

Anderson LW, Krathwohl DR (2001) A taxonomy for learning, teaching, and assessing: a revision of bloom's taxonomy of educational objectives. Longman, New York

Ante L (2021) Smart contracts on the blockchain-A bibliometric analysis and review. Telematics Informatics 57:101519. https://doi.org/10.1016/j.tele.2020.101519

Asarta CJ, Bento R, Fornaciari CJ, Dean KL, Arbaugh JB, Hwang A (2018) The scholarship of teaching and learning: changing the dominant narrative about (and in) research institutions. J Manag Educ 42:731-748. https://doi.org/10.1177/1052562918777271

Bagloee SA, Esumati M, Dia H, Ghaderi H, Pettit C, Asadi M (2021) Blockchain: the operating system of smart cities. Cities 112:103104. https://doi.org/10.1016/j.cities.2021.103104

Bartsch F, Neidhardt N, Nüttgens M, Holland M, Kompf M (2018) Anwendungsszenarien für die Blockchain-Technologie in der Industrie 4.0. HMD 55(6):1274-1284. https://doi.org/10.1365/s40702-01800456-8

Bertelsmann Stiftung (2017) Inclusive Growth for Germany 18: Technological Innovation and Inclusive Growth in Germany. https://www.bertelsmann-stiftung.de/en/publications/publication/did/inclusivegrowth-for-germany-18-technological-innovation-and-inclusive-growth-for-germany/. Zugegriffen: 30. Nov. 2021

Biggs JB, Tang C (2011) Teaching for quality learning at university: what the student does. McGraw-Hill, Glasgow

Büttgen M, Dicenta J, Spohrer K, Venkatesh V, Raman R, Hoehle H, De Kayser A, Verbeeck C, Zwienenberg T, Jørgensen K, Beck R, Rikken O, Janssen M, Kwee Z, Schär F (2021) Blockchain in service management and service research-developing a research agenda and managerial implications. J Serv Manag Res 5(2):71-102. https://doi.org/10.15358/2511-8676-2021-2-71

Commission EU (2005) Mobilising the brainpower of europe: enabling universities to make their full contribution to the lisbon strategy. Communication from the commission. http://aei.pitt.edu/42884/1/ com2005_0152.pdf. Zugegriffen: 21. Sept. 2021

Crosby M, Nachiappan Pattanayak P, Verma S, Kalyanaraman V (2016) Blockchain technology: beyond Bitcoin. Appl Innov Rev 2(2):5-19 
European Institute of Innovation and Technology (2012) Catalysing innovation in the knowledge triangle. Publication for the EIT. https://eit.europa.eu/sites/default/files/EIT_publication_Final.pdf. Zugegriffen: 21. Sept. 2021

Fragkandreas T (2017) Innovation paradoxes: a review and typology of explanations. Prometheus 35:267-290. https://doi.org/10.1080/08109028.2018.1506620

Halaburda H, Levina N, Min S (2019) Understanding smart contracts as a new option in transaction cost economics. Proceeding of the Fortieth International Conference on Information Systems (ICIS).

Kibler S, Eckardt L (2018) On the role of tasks in virtual game-based learning: the example of lost in Antarctica. Proceedings der International Association of University Libraries (IATUL).

Kumar V, Ramachandran D, Kumar B (2021) Influence of new-age technologies on marketing: a research agenda. J Bus Res 125:864-877. https://doi.org/10.1016/j.jbusres.2020.01.007

Lacity M, van Hoek R (2021) What we've learned so far about Blockchain for business. MIT Sloan Manage Rev 62(3):47-54

Lassnigg L, Hartl J, Unger M, Schwarzenbacher I (2017) Higher education institutions and knowledge triangle: improving the interaction between education, research and innovation. HIS working paper. https://irihs.ihs.ac.at/id/eprint/4228/1/rs118.pdf. Zugegriffen: 21. Sept. 2021

Lipmanowicz H, McCandless K (2014) The surprising power of liberating structures: simple rules to unleash A culture of innovation. Liberating Structures Press, Seattle

Meier A, Stormer H (2018) Blockchain = distributed ledger + consensus. HMD 55(6):1139-1154. https:// doi.org/10.1365/s40702-018-00457-7

Miller K, McAdam R, McAdam M (2018) A systematic literature review of university technology transfer from a quadruple helix perspective: toward a research agenda. R D Manag 48:7-24. https://doi.org/ 10.1111/radm. 12228

Moher D, Liberati A, Tetzla J, Altman DG, the PRISMA Group (2010) Preferred reporting items for systematic reviews and meta-analyses: the PRISMA statement. J Surg 8(5):336-341. https://doi.org/ 10.1371/journal.pmed.1000097

Nagel E, Kranz J, Sandner P, Hopf S (2019) How Blockchain facilitates smart city applications-development of a multi-layer taxonomy. Proceedings of the 27th European Conference on Information Systems (ECIS).

Oliveira L, Zavolokina L, Bauer I, Schwabe G (2018) To token or not to token: tools for understanding Blockchain tokens. Proceedings Thirty Ninth International Conference on Information Systems (ICIS).

Patton MQ (1990) Qualitative evaluation and research methods. SAGE, Hoboken

Phan PH, Siegel DS, Wright M (2009) New developments in technology management education: background issues, program initiatives, and a research agenda. Acad Manag Learn Educ 8:324-336. https://doi.org/10.5465/AMLE.2009.44287934

Pilkington M (2016) Blockchain technology: principles and applications. In: Olleros FX, Zhegu M (Hrsg) Research handbook on digital transformations. Edward Elgar, Cheltenham Northampton, S 225-251

Potočnik J (2006) Embedding European science into European society. Austrian Academy of Sciences,

Reddy N, Zhao L (1990) International technology transfer: a review. Res Policy 19:285-307. https://doi. org/10.1016/0048-7333(90)90015-X

Sarma SE, Bonville WB (2020) Applying New Education Technologies to Meet Workforce Education Needs. https://workofthefuture.mit.edu/wp-content/uploads/2020/10/2020-Research-Brief-SarmaBonvillian.pdf. Zugegriffen: 30. Nov. 2021

Schütz AE, Fertig T, Weber K, Vu H, Hirth M, Tran-Gia T (2018) Vertrauen ist gut, Blockchain ist besser - Einsatzmöglichkeiten von Blockchain für Vertrauensprobleme im Crowdsourcing. HMD 55(6):1155-1166. https://doi.org/10.1365/s40702-018-00471-9

Treiblmaier H (2021) The token economy as a key driver for tourism: entering the next phase of blockchain research. Ann Tour Res 91:103177. https://doi.org/10.1016/j.annals.2021.103177

Unger M, Marsan GA, Meissner D, Polt W, Cervantes M (2020) New challenges for universities in the knowledge triangle. J Technol Transf 45:806-819. https://doi.org/10.1007/s10961-018-9699-8

Weiblen T, Chesbrough HW (2015) Engaging with startups to enhance corporate innovation. Calif Manage Rev 57:66-90. https://doi.org/10.1525/cmr.2015.57.2.66

Whitaker J, New JR, Ireland RD (2016) MOOCs and the online delivery of business education what's new? What's not? What now? AMLE 15:345-365. https://doi.org/10.5465/amle.2013.0021 